\title{
Antidiabetic agents and vascular events
}

\author{
H. KEEN
}

From the Unit for Metabolic Medicine, Department of Medicine, Guy's Hospital, London

The physician concerned with the treatment of diabetic patients has increasingly been called upon in recent years to define his 'aims of treatment'. He has a hierarchy of aims. The preservation of life for the juvenile-type, insulin-dependent diabetic is an obvious imperative and has been achieved. Relief of symptoms is the next clear aim and this is followed by the goal of 'metabolic normalization', often interpreted as the return of blood glucose levels to the physiological range. Beyond these comparatively well defined, short-term goals and entangled with them lie more vague and distant objectives. They include the avoidance, or at least the postponement, of the long-term 'complications' of diabetes, the retinopathy which ranks foremost as a cause of blindness in the middle-aged, renal failure which contributes to the early death, particularly of juvenile diabetics, and neuropathy responsible for much pain and chronic disability in the diabetic population. In a separate but equally menacing category, is the twoto threefold excess of morbidity due to disease of large and medium-sized arteries in diabetic men and the sixfold excess in diabetic women.These objectives of treatment are embraced within the general aim of prolonging the length and enhancing the quality of the life of the diabetic.

No such exposition will be allowed to pass without criticism. A strong body of opinion (Siperstein, Unger, and Madison, 1968; Siperstein, 1972) holds that the 'complications' of diabetes are not, in fact, complications at all but a disorder of vascular basement membrane largely independent of the metabolic disorder, maybe preceding and perhaps even responsible for it, a view which is hotly contested on technical (Kilo, Vogler, and Williamson, 1972; Williamson, Vogler, and Kilo, 1973; Østerby, 1972) and experimental (Bloodworth, Engerman, and Anderson, 1973) grounds. Even assuming that the length of life could be increased by rigid adherence to a strict regime of treatment, would this so affect the quality of those dearly bought years as to make one question the price? The demands of really rigid control seek out and enhance many personality flaws in the young. Even for the maturity-onset type of diabetic the restrictions on dietary freedom, the extra cost of the regimen and the possibility of the need for daily insulin injections is a burden only justified by the clear prospect of some long-term benefit.

It is against this background that one must view the advent almost 20 years ago of the oral antidiabetic agents. Stemming from the chance observation in 1942, in occupied France, of hypoglycaemia occurring in patients treated with a new sulphonamide (Loubatières, 1957) a large family of sulphonylurea derivatives has found its way into clinical practice. Many more are waiting in the wings. Oral guanidine derivatives (Watanabe, 1918), more eclipsed by the arrival of insulin than dropped because of toxicity in the 20 s, reappeared as the biguanides now in wide clinical use (Krall and CameriniDavalos, 1957). These oral antidiabetic agents seemed to assist in fulfilling at least some of the aims of treatment. They relieved symptoms and lowered blood glucose in a high proportion of noninsulin-dependent diabetics. They improved the quality of the diabetic life by relieving some of the need for insulin injections. In some parts of the world, and increasingly with the years, they were used almost as a substitute for dietary restriction. But as with insulin treatment and even with dietary restriction itself, it was unclear whether the use of oral antidiabetic agents helped in achieving the more distant aims of prolonging life and reducing vascular disease.

At the crudest level of evaluation, we can say as clinicians that diabetic retinopathy and coronary disease have certainly not been abolished from our clinics over the last 20 years. The cohort study of new diabetics, begun in Birmingham in 1948 and reported up to 1968 at the spring 1973 meeting of the British Diabetic Association (Fitzgerald and Malins, 1973), suggested a progressive reduction in excess mortality of diabetics over non-diabetics since the era of introduction of the oral agents, but this was a continuation of a preceding improvement, perhaps due to other causes. National mortality figures for diabetes are more difficult to interpret in this respect. Reid and Grimley Evans (1970) have drawn attention to an arrest in the late 1950 s in the previous steady decline in rates of mortality certified as due to diabetes, with even an indication of a subsequent up- 
turn in rates in males. However, such figures may merely reflect an increasing incidence (or increasing awareness) of diabetes. They may also suggest that fewer diabetics are dying of, and being certified as deaths due to, other causes, such as coronary disease, and so appearing in the category of death due to diabetes (Warren and Corfield, 1973).

\section{The Bedford Tolbutamide Trial}

There are great problems in trying to evaluate the essentially preventive effects of treatment on the long-term course of diabetes mellitus. Any attempt at evaluation whether retrospective or prospective on a group of patients who have been allocated to treatment categories other than randomly must raise the question of whether differences in outcome between treatment groups was truly an effect of the different treatments or a reflection of the reasons for which particular patients were selected for different treatments (or an interaction between the two). In such cases there is no way of separating the effects of the treatment from the effects of the disease. Furthermore, if the form of the treatment is known to the clinician, it may influence the interpretation of symptoms or signs presented by the patient'dizziness' in a diabetic might well be attributed to hypoglycaemia were he on insulin but more likely to 'cerebrovascular disease' were he treated by diet alone. There are also special problems of clinical trials in which prevention is the endpoint, particularly when the endpoints have a subjective quality to them, eg, the chest pain of coronary disease, socalled 'soft endpoints', but also even when the endpoints are 'hard' as with death, when it comes to attributing a cause. Thus, in order to make a valid judgment of the influence of oral antidiabetic agents on vascular events we require special conditions, including random allocation of suitable patients to different treatments, preferably including a placebo group, ignorance of the investigator of the form of treatment used, prospective study with predetermined and fixed criteria for endpoints and adequate numbers in treatment groups to allow statistically valid assertions to be made regarding treatment effects or the much more costly assertion of an absence of effect.

It was with some of these considerations in mind that in 1962 we committed ourselves to a long-term trial of tolbutamide in mild diabetics found in the course of the Bedford diagnostic survey (Sharp, Butterfield, and Keen, 1964). The analysis of the Bedford follow-up data has presented us with a number of formidable difficulties and we have, so far, overcome only a proportion of them. The basic intention in the Bedford study was to submit a group of borderline diabetics, defined on the basis of a single measurement of blood sugar made two hours after a $50 \mathrm{~g}$ glucose load, to a long-term, randomized, double-blind trial of tolbutamide versus placebo, superimposing on each of these groups, randomized allocation to true carbohydrate restriction $(120 \mathrm{~g} /$ day) or nominal table sugar reduction. At regular six monthly visits we planned to follow the evolution of the metabolic disorder by repeated standardized measurement of blood sugar levels, and also to record the appearance of the systemic accompaniments of diabetes in the eyes, the kidneys, the peripheral nerves and the cardiovascular system by the repeated application of standardized tests and investigations. In all such evaluations, the double-blind structure of the trial has been faithfully maintained, the code being broken only when it was clearly in the subject's interest to do so, that is, in the case of serious illness or when requested by professional colleagues. Sets of conditions were defined which terminated the subject's stay in the trial and which consisted largely of clear evidence of metabolic deterioration. Since data were computerized, it was possible to make interim analyses of progress by treatment group without revealing the form of treatment for individual subjects to the investigator.

Most of the data analysis to the present has concerned the advent of new cardiovascular events; early comparisons after five years (Keen, Jarrett, Chlouverakis, and Boyns, 1968) suggested that dietary recommendation per se had little effect on these events and attention has largely been concentrated on comparisons of tolbutamide versus placebo treatment. The cardiovascular events recorded were defined and varied from, at the mildest end of the scale, a new, Minnesota-codable ECG deterioration, through 'WHO-questionnaire-positive' history of arterial disease symptoms, to death certified by the attending physician or at necropsy as cardiovascular in cause (Rose and Blackburn, 1968).

At the five-year and seven-year (Keen and Jarrett, 1970) time points, we were thus able to compare the number of individuals in tolbutamide and placebo treatment groups suffering one or other (or multiples) of these 'index events'. At five years, $22 \%$ of the tolbutamide-treated subjects had sustained such an event, significantly fewer $(P<0.05)$ than the $33 \%$ in the placebo group. At seven years the rates were $28 \%$ and $38 \%$ respectively $(0.1>\mathrm{P}>0.05)$, and further analysis suggested that it was with the younger, more hyperglycaemic subjects who entered the trial without hypertension or prior history of arterial disease that the apparent tolbutamide advantage predominantly lay. It was only when total numbers of individuals with one or other of the variety of events were compared that significant 
differences could be demonstrated. Numbers of individuals experiencing single classes of event were too few to distinguish an effect of the drug from the play of chance, though trends favoured tolbutamide treatment in almost all individual classes of vascular events.

\section{University Group Diabetes Programme}

In 1970 the unexpected and controversial findings of the University Group Diabetes Program in the United States were published (University Group Diabetes Program, 1970, I and II) claiming to have demonstrated a significant excess of 'cardiovascular deaths' in asymptomatic, maturity-onset diabetics treated with tolbutamide compared with those treated with a placebo. Few clinical trials can have been subjected to such detailed scrutiny in an atmosphere which has on occasions been more gladiatorial than scientific. The most recent (though not necessarily the final) verdict on this historic controversy has been handed down in the Report of a Special Committee of the Biometric Society (Committee for the Assessment of Biometric Aspects of Controlled Trials of Hypoglycemic Agents, 1975) which largely confined its attention to the question of mortality and in whose opinion '. . . the UGDP trial has raised suspicions that cannot be dismissed on the basis of other evidence presently available'. Others have taken different views of the published results, and it is difficult for the clinician to understand why the two- and one-half-fold increase in cardiovascular death (a category probably accounting for most deaths in western diabetic populations) should have failed to cause a statistically significant increase in total death rates in the UGDP tolbutamide group. This was perhaps partly explained by the spectacularly low rate of deaths attributed to coronary disease in the placebo group, not one of the 21 deaths in that group apparently satisfying UGDP criteria for myocardial infarction, a circumstance evidently less disturbing to the biostatistician than to the clinician and one to be set alongside the Biometric Society's views that 'the possibility that deaths may have been allocated to cardiovascular causes preferentially in the groups receiving oral therapy exists; and, in view of the "non-significance" of differences in total mortality, some reservation, about the conclusion that the oral hypoglycaemics are toxic must remain'.

\section{Bedford Analysis at Eight and a Half Years}

The UGDP findings prompted us (Keen, Jarrett, and Fuller, 1974) to re-examine the experience of the Bedford subjects once again at the time point of eight and a half years. First, we made our comparisons, as in past analyses, using chi-square analysis of treatment effects on the accumulated numbers of individuals with specified events or combinations of events, comparable to methods used by the UGDP, in assessing the effects of treatment on mortality experience.

Our attention had been drawn to the powerful new statistical technique, the log rank test procedure, developed and applied in the Department of the Regius Professor of Medicine at Oxford (Peto and Pike, 1973) for the purpose of making continuing evaluation of the effects of treatment on malignant disease, a modified cumulative life-table method which computes the probability that 'time to failure' differs between treatments. It was possible to adapt this technique to our problem, largely thanks to the special computer skills of my colleague, Dr John $\mathrm{H}$. Fuller, and the generous assistance of Dr R. Peto and Professor Sir Richard Doll, who are, of course, in no sense responsible for the use to which we have put the technique, nor the inferences we have drawn from the results of its use. The use of the log rank test procedure computer program enabled us to evaluate the effects of treatment in the borderline diabetic group as a whole, or within defined sets and subsets of subjects, eg, under and over 60 at entry to trial; male and female; top, middle or bottom tertile of initial blood sugar distribution; tertile of ponderal index, taking for treatment comparisons, the time to death or the time to other specified arterial events or combinations of events.

\section{Mortality and Morbidity}

The simple layout of deaths by selected causal categories in the two treatment groups occurring during the eight and a half years to 1 January 1971 is shown in table $I$. The presentation is selected to resemble that of the UGDP (University Group Diabetes Program, 1970, II). There is clearly evidence neither of advantage nor of deleterious effect of tolbutamide in respect of total or of cardiovascular mortality. To this extent, while our findings in Bedford cannot be said to refute the assertion of the UGDP, they offer it no shred of support. When this simple comparison of eight and a half-year totals was directed to items of cardiovascular morbidity (angina or infarct or ECG event) in those under 60 at recruitment to trial, $23 \%$ of 74 tolbutamidetreated subjects had experienced one or another of these events compared with $41 \%$ of 61 placebotreated subjects $(P<0.05)$. The mean ages $( \pm S E)$ in these groups (tolbutamide 46.6 years $\pm 1 \cdot 2$; placebo 45.7 years \pm 1.3 ) were very similar. The advantage to these tolbutamide-treated subjects was even more significant when comparison of event rates in this age group was limited to subjects in the highest 


\begin{tabular}{lcc}
\hline & Placebo & Tolbutamide \\
\hline Number at risk of death & 125 (M68 F57) & 123 (M61 F62) \\
Cardiovascular causes & 9 & 10 \\
$\quad$ Myocardial infarction & 0 & 0 \\
Sudden death & 4 & 4 \\
Other heart disease & 6 & 4 \\
Extracardiac heart disease & 19 & 3 \\
All cardiovascular causes & 4 & 4 \\
Non-cardiovascular causes & 4 & 25 \\
Cancer & 27 & $14 \cdot 6$ \\
Other causes & $15 \cdot 2$ & $20 \cdot 3$ \\
All causes & $21 \cdot 6$ & \\
Carcentage dead from: & & \\
All causes & & \\
\hline
\end{tabular}

Table I Mortality after eight and a half years in Bedford tolbutamide trial

${ }^{1}$ Deaths by certified cause to 1 January 1971 .

tertile of the blood sugar distribution (tolbutamide $25.0 \%$; placebo $76.5 \%, P<0.01$ ). Statistically significant advantage $(P<0.05)$ to the tolbutamidetreated group was also shown for the under 60 s and for all ages for the event combination angina or infarct or ECG event or coronary death and for the event combination ECG event or cardiovascular event or cardiovascular death. In only one comparison was there a statistically significant advantage for placebo over tolbutamide and this was for the event combination ECG event or cardiovascular event or cardiovascular death in the over 60 s in the lowest blood sugar tertile. However, tolbutamidetreated subjects in this subgroup entered the trial with a much higher prevalence of preexisting arterial disease $(45.5 \%)$ and of hypertension $(54.5 \%)$ than the placebo-treated subjects $(0 \%$ and $23 \cdot 1 \%$ respectively).

Application of the log rank test procedure to the individuals or specified subsets of individuals sustaining an event or one or another of a specified set of events is a very rigorous test of assertions of difference. The chief reason for this is the exclusion from consideration of any individual from the time he suffers an event, defaults from a follow-up examination or leaves the group by 'becoming diabetic' by our present criteria. Unlike simple chi-square comparisons which take as $n$ the whole starting group, the censoring described above leads to a rapidly dwindling $n$ and the consequent need for much larger treatment differences to achieve statistical significance. Of the large number of such comparisons made, the only differences achieving technical levels of significance $(P<0.05)$ showed an advantage for tolbutamide takers in the top tertile of the initial blood sugar distribution in respect of the event combination cardiovascular event or cardiovascular death or ECG change and for the event combination angina or infarct or coronary death or ECG change. It is unnecessary to point out the considerable overlap and so the absence of independence of these two combinations of events. The results of this eight and a half-year analysis have been published in full (Keen et al, 1974) with our conclusion that they were compatible with our earlier findings, that the hypothesis of a cardiovascular protective action of tolbutamide in borderline diabetics receives stronger support from simple chi-square comparisons than from the more rigorous log rank procedure and that even this later method offered a little support for the hypothesis and none against it.

\section{Phenformin-UGDP Findings}

An evaluation of the effects of phenformin, $50 \mathrm{mg}$ twice daily, was introduced late into the UGDP trial design in only six of the 12 cooperating centres. In order to achieve numbers of phenformin-treated patients comparable to those of the other treatment groups, approximately three patients were assigned to phenformin for one to each of the other treatments. This distortion of the design made comparisons rather less 'tidy' than in the case of tolbutamide, providing a very small contemporaneous placebo group for comparison and leading to the use of insulin-injecting groups with and without the small placebo group added in for comparative purposes. A preliminary report (University Group Diabetes Program, 1971) suggested that mortality rates, both total and cardiovascular in the phenformin group compared unfavourably with the selected contrast groups mentioned above. Very recently these findings have been published in extenso (University Group Diabetes Program, 1975). The initial conclusions have been sustained and no evidence found that the excess mortality in the phenformin group could be accounted for by chance inclusion of 'sicker' 
diabetics in this group at the start, at any rate in respect of those characteristics which were measured at baseline. The report draws attention to an elevation of blood pressure and pulse rate in phenformintreated patients, compared with the contrast groups, a new finding which, it is considered, may contribute to the explanation of the increased mortality.

\section{Whitehall Phenformin Trial}

Since 1967 we have been conducting a randomized double-blind placebo-controlled trial of phenformin $(50 \mathrm{mg} /$ day timed disintegration capsule) in 204 borderline diabetics newly detected during a screening survey of London-based civil servants aged $\mathbf{4 0}$ years or more recruited into the trial over a two and a half-year period (Reid, Brett, Hamilton, Jarrett, Keen, and Rose, 1974). Much along the same lines as in Bedford half of each of the two treatment groups have also been randomly assigned to restricted carbohydrate diets $(120 \mathrm{~g} /$ day $)$ and standardized data have been collected at regular six-monthly visits to a follow-up clinic. Failed attendances have been exhaustively investigated, with careful monitoring for mortality (of which we are also promptly informed from central sources). Thirteen deaths had been recorded after a mean of approximately five years of follow up, and their distribution by phenformin or placebo treatment is shown in table II. No differential mortality by treatment is discernible either in totals or causal groups.

We have also compared the mean systolic and diastolic (fourth phase-sudden change of note of Korotkow sounds) arterial pressure levels in a cohort of those subjects attending all of the first six follow-up clinics (two and a half years) of the study. Medication (placebo or control) was stopped a week before each of the visits except the fifth, on which occasion medication was included up to and including the day of the visit. On this occasion a standard electrocardiogram was also recorded, and

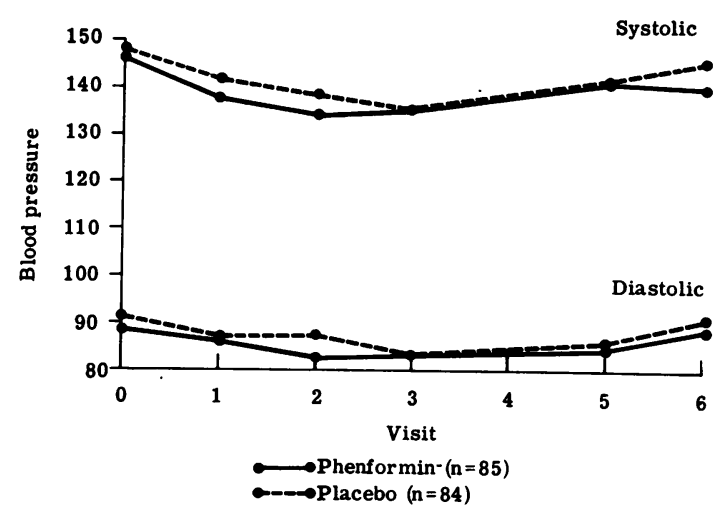

Figure Mean systolic and diastolic (phase 4) blood pressure values in a cohort of borderline diabetic subjects, all males aged 40 or more, 85 treated with phenformin (50 mg/day \pm carbohydrate restriction), and 84 treated with placebo ( \pm carbohydrate restriction). $V$ isit 0 represents the initial survey observation before assignment to treatment. Visits 1-6 were at six-monthly intervals. Treatment was started immediately after visit 1, withdrawn one week before each of the subsequent visits except visit 5 when it was continued up to and including the day of the visit. Cohort is of "no missed visit' subjects.

from this measurements were made of the mean beat-to-beat interval for each subject. Similar measurements were made from electrocardiograms for the same subjects recorded at the survey visit and before assignment to treatment groups. All measurements and calculations were made in ignorance of the treatment assignments.

The figure shows the absence of any significant difference in mean arterial pressure between placeboand phenformin-treated subjects.

Table III shows the mean beat-to-beat interval at baseline and at the fifth follow-up visit in subjects assigned to placebo and phenformin treatment groups. There are no differences either between occasions or between treatments.

\begin{tabular}{llc}
\hline & \multicolumn{2}{c}{ Deaths by Certified Cause at 5 years } \\
\cline { 2 - 3 } & Placebo & Phenformin \\
\hline Number at risk of death & 98 & 106 \\
Mean age at recruitment & $57 \cdot 0$ & $56 \cdot 5$ \\
Number of deaths & 6 & $7 \cdot 7$ \\
Mean age of dead 1) at recruitment & $58 \cdot 0$ & $58 \cdot 7$ \\
Certified causes of death & $60 \cdot 0$ & $62 \cdot 3$ \\
Myocardial infarction & 3 & 3 \\
Other heart disease & 0 & 2 \\
Stroke/subarachnoid haemorrhage & 1 & 1 \\
Cancer & 1 & 1 \\
Other cause & 1 & 0 \\
\hline
\end{tabular}

Table II Mortality after five years in Whitehall phenformin trial 


\begin{tabular}{lll}
\hline & Placebo & Phenformin \\
\hline Baseline & $20.26 \pm 0.47$ & $20.35 \pm 0.42$ \\
Fifth visit & $21.09 \pm 0.46$ & $20.69 \pm 0.41$ \\
\hline
\end{tabular}

Table III Mean beat-to-beat interval at baseline and fifth visit in placebo and phenformin groups ${ }^{1}$ of Whitehall phenformin trial

${ }^{1} R$ peak to $R$ peak interval in $\mathrm{mm}$ at standard rate; mean $\pm S D$.

Other investigations involving patients receiving long-term phenformin therapy on a trial basis are in progress (Danowski et al, 1974; Feldman, Crawford, Elashoff, and Glass, 1973; Tzagournis and Reynertson, 1972) and their findings in respect of blood pressure and pulse rate effects are awaited with interest. No excess mortality is indicated in the published reports of Feldman and of Tzagournis.

\section{Conclusions}

This account has perhaps done little more than to draw attention to the change in the attitude to oral antidiabetic agents over the last five years, to indicate the reasons for the change, and to describe the present contradictory state of the evidence. The assumption that oral agents were almost wholly benign has inevitably been challenged, and the challenge has equally predictably been resisted. Much of the argument has centred on the effects of tolbutamide and phenformin on cardiovascular disease in diabetics and in particular on death attributed to cardiovascular cause. The assertion of the increased hazard of cardiovascular death made by the UGDP in respect of tolbutamide and phenformin treatment is not supported by other independent randomized studies, there are hints that tolbutamide may confer some protection against non-fatal disease of large vessels and there is no evidence to the contrary. Direct comparisons between trials is difficult for they differ in respect of the type of subjects studied, the dose of medication used, the methods of surveillance employed and the variety of endpoints selected. There are economic and organizational problems inherent in any long-term trial where the endpoint is preventive, when success is to be measured by the failure of some expected event to occur rather than the 'cure' of some existing condition. Just how far one can generalize from the two particular oral agents to all other oral agents is questionable. Measurement of 'costs' of treatment has been considerably more thorough than the estimate of 'benefits' in assessing the role of oral agents in the therapy of diabetes mellitus. However, the controversy is settled, if indeed it is ever finally settled, one cannot but welcome the new perspectives and new approaches to the evaluation of long-term therapy in general. Perhaps physicians, the regulatory agencies and the pharmaceutical industry should be giving more thought, individually and jointly, to creating suitable machinery to meet an increasing challenge.

\section{References}

Bloodworth, J. M. B., Jr., Engerman, R. L., and Anderson, P. J. (1973). Microangiopathy in the experimentally diabetic animal. In Vascular and Neurological Changes in Early Diabetes, edited, by R. A. Camerini-Dávalos and H. S. Cole, p. 245. Academic Press, New York.

Committee for the Assessment of Biometric Aspects of Controlled Trials of Hypoglycemic Agents (1975). Report. J. Amer. med. Ass., 231, 583-608.

Danowski, T. S., Sunder, J. H., Stephan, T., Nolan, S., Ohlsen, P. M. Ahmad, U., Vidalon, C., and Wingert, J. P. (1974). Phenformin therapy of chemical diabetes. J. clin. Pharmacol., 14, 638-650.

Feldman, R., Crawford, D., Elashoff, R., and Glass, A. (1973). Progress report on the prophylactic use of oral hypoglycaemic drugs in asymptomatic diabetes: neurovascular studies. In Vascular and Neurological Changes in Early Diabetes, edited by R. A. Camerini-Davalos and H. S. Cole, p. 557. Academic Press, New York.

Fitzgerald, M. G., and Malins, J. M. (1973). Diabetic mortality in Birmingham 1945-1965: results of a further 5 year follow-up presented to the Medical and Scientific section of the British Diabetic Association, Spring 1973.

Keen, H., and Jarrett, R. J. (1970). Glucose tolerance, plasma lipids and atherosclerosis. In Atherosclerosis: Proceedings of the 2nd International Symposium, edited by R. J. Jones, p. 435 . Springer, New York.

Keen, H., Jarrett, R. J., Chlouverakis, C., and Boyns, D. R. (1968). The effect of treatment of moderate hyperglycaemia on the incidence of arterial disease. Postgrad. med. J., 44, 960-965.

Keen, H., Jarrett, R. J., and Fuller, J. H. (1974). Tolbutamide and arterial disease in borderline diabetics. In Diabetes: Proceedings of the Eighth Congress of the International Diabetes Federation, edited by W. J. Malaisse, J. Pirart, and J. Vallence-Owen, p. 588. Excerpta Medica, Amsterdam.

Kilo, C., Vogler, N., and Williamson, J. R. (1972). Muscle capillary basement membrane changes relating to aging and to diabetes mellitus. Diabetes, 21, 881-905.

Krall, L. P., and Camerini-Davalos, R. (1957). Early clinical evaluation of a new oral non-sulfonylurea hypoglycemic agent. Proc. Soc. exp. Biol. (N.Y.), 95, 345-347.

Loubatières, A. (1957). The hypoglycemic sulfonamides: history and development of the problem from 1942 to 1955. Ann. N.Y. Acad. Sci., 71, 4-11.

Østerby, R. (1972). Morphometric studies of the peripheral glomerular basement membrane in early juvenile diabetes 1 . Development of initial basement membrane thickening. Diabetologia, 8, 84-92.

Peto, R., and Pike, M. C. (1973) Conservation of the approximation $\Sigma(\mathrm{O}-\mathrm{E})^{2} / \mathrm{E}$ in the logrank test for survival data or tumour incidence data. Biometrics, 29, 579-588.

Reid, D. D., Brett, G. Z., Hamilton, P. J. S., Jarrett, R. J., Keen, H., and Rose, G. (1974). Cardiorespiratory disease and diabetes among middle-aged male Civil servants: a study of screening and intervention. Lancet, 1, 469-473.

Reid, D. D., and Grimley Evans, J. (1970). New drugs and changing mortality from non-infectious disease in England and Wales. Brit. med. Bull., 26, 191-196.

Rose, G. A., and Blackburn, H. (1968). Cardiovascular Survey Methods. World Health Organization, Geneva.

Sharp, C. L., Butterfield, W. J. H., and Keen, H. (1964). Diabetes survey in Bedford 1962. Proc. roy. Soc. Med., 57, 193.

Siperstein, M. D., Unger, R. H., and Madison, L. L. (1968). Studies of muscle capillary basement membranes in normal subjects, diabetic and prediabetic patients. J. clin. Invest., 47, 1973-1999.

Siperstein, M. D. (1972). Capillary basement membrane and diabetic microangiopathy. Advanc. intern. Med., 18, 325-344.

Tzagournis, M., and Reynertson, R. (1972). Mortality from coronary heart disease during phenformin therapy. Ann. intern. Med., 76, 587-592. 
University Group Diabetes Program (1970). A study of the effects of hypoglycemic agents in vascular complications in patients with adult-onset diabetes. I. Design, methods and baseline results. II. Mortality results. Diabetes, 19, Suppl. 2, 747-783 and 789-800.

University Group Diabetes Program (1971). III. Clinical implications of UGDP results. IV. A preliminary report on phenformin results. J. Amer. med. Ass., 218, 1400-1410 and 217, 777-784.

University Group Diabetes Program (1975). V. Evaluation of phenformin therapy. Diabetes, 24, 65-184.

Warren, M. D., and Corfield, A. (1973). Mortality from diabetes.
(Letter). Lancet, 1, 1511-1512.

Watanabe, C. K. (1918). Studies in the metabolic changes induced by the administration of guanidine bases. 1 Influence of injected guanidine hydrochloride upon blood sugar content. J. biol. Chem., 33, 253-265.

Williamson, J. R., Vogler, N. J., and Kilo, C. (1973). Early capillary basement membrane changes in subjects with diabetes mellitus. In Vascular and Neurological Changes in Early Diabetes, edited by R. A. Camerini-Dávalos and H. S. Cole, p. 363. Academic Press, New York. 